95\% CI 1.07-1.53), reporting vaginal sex (OR 5.89; 95\% CI 2.42-14.36), non-penetrative sex (OR 3.47; 95\% CI 1.56-7.75), alcohol use (OR 3.34; 95\% CI 1.26-8.87), smoking (OR 5.82; 95\% CI 1.12-30.37), chewing khat (OR 3.19; 95\% CI 1.278.01 ), and being at university rather than high school (OR 4.58; 95\% CI 1.94-10.79) were associated with increased likelihood of STI ( $\mathrm{p} \leq 0.05$ ). Condom use was protective (OR 0.32; 95\% CI 0.11-0.94).

Conclusion There was considerable demand for STI screening, and a substantial burden of STIs was observed. These findings support the need for reproductive health interventions to reduce adolescent risk for STIs, pregnancy, and their complications.

Disclosure of interest statement This research was funded by a 2011 developmental grant from the University of Washington Global Centre for Integrated Health of Women, Adolescents, and Children (Global WACh).

\section{P03.20 "HIGH RISK FACTORS AND THEIR ASSOCIATION WITH SEXUALLY TRANSMITTED INFECTIONS AMONG ASYMPTOMATIC PREGNANT ADOLESCENTS"}

${ }^{1}$ Pratima Mittal ${ }^{*}$, ${ }^{1}$ Priti Ghope, ${ }^{1}$ Rekha Bharti, ${ }^{2}$ Subhash C Sonkar, ${ }^{1}$ Anita Kumar, 2Daman Saluja. 'Department of Ob-Gyn, Vaedhman Mahavir Medical College \& Safdarjung Hospital New Delhi, India; ${ }^{2}$ Dr. B. R. Ambedkar Center for Biomedical Research, University of Delhi, Delhi-110007, India

\subsection{6/sextrans-2015-052270.248}

Background Adolescents are at high risk for sexually transmitted infections (STIs) because of their high risk behaviour and physiological susceptibility. The present study aimed to find out prevalence of high risk factors and their association with STIs among asymptomatic pregnant adolescents.

Methods This was a cohort study conducted in the Department of Obstetrics and Gynaecology, Vardhman Medical College and Safdarjung hospital in association with Dr B. R. Ambedkar Centre for Biomedical Research, University of Delhi. A total of 320 asymptomatic adolescent pregnant women willing to participate in the study protocol were enrolled. A detailed history including sexual, marital, obstetric, personal and clinical details was taken. After clinical examination, cervical and high vaginal swabs were collected for testing by PCR technique for Chlamydia Trachomatis, Neisseria Gonorrhoea and Trichomonas Vaginalis infections. The data hence collected was analysed to find out prevalence of high risk factors and their association with STIs.

Results Out of 320 women enrolled in the study, 41 (12.81\%) tested positive for one of the 3 STIs tested. Most of the subjects were 19 years old $(76.56 \%)$, belonged to urban area $(80.94 \%)$, were from low income group (82.81\%), had not been to high school $(70 \%)$ and $78.13 \%$ had at least one high risk factor for STIs. Among the high risk factors for STIs studied, $31.87 \%$ had age $<18$ years at the time of first sexual intercourse, $75.94 \%$ had never used any contraception, $1.87 \%$ had history of tobacco or alcohol use, $7.81 \%$ had past history of vaginal discharge. Out of these high risk factors for STIs only past history of vaginal discharge was significantly associated with presence of STIs, $\mathrm{p}=$ 0.0001 .

Conclusion The high risk factors for STIs are present in more than $3 / 4^{\text {th }}$ of pregnant adolescent women and past history of vaginal discharge is significantly associated with STIs.

\section{P03.21 BE SAFE. STAY WELL: FOUR VIDEOS TO EDUCATE INTERNATIONAL STUDENTS ON SEXUAL HEALTH AND STAYING SAFE IN AUSTRALIA}

M Roberts, K Kerry*. Communicable Disease Control Directorate, Department of Health WA

10.1136/sextrans-2015-052270.249

Background In recent years there has been a considerable growth in the international student population in Australia driven primarily by university study and vocational training. Studying and living in a new country can bring a range of social challenges, and international students have shown to have limited understanding on sexual health within an Australian context. Following consultation and focus testing with international students and health professionals, the Sexual Health and Bloodborne Virus Program (SHBBVP) have developed a collection of videos titled "Be Safe Stay Well" aimed at providing students with an understanding of sexual health and the health care system in WA.

Methods Three focus groups were conducted with a total of 36 international students representing 22 different countries. Students reported wanting to know more about sexual health, safe sex and STIs. It was identified that having a resource providing sexual health information would be useful for the target group. In collaboration with the WA Department of Health's Communications Directorate and an external production company, the four videos titled "Be Safe. Stay Well" were developed.

Results The videos use animation and kinetic typography to appeal to an international audience whose first language is not usually English. They have been spoken by young people from a range of countries to ensure authenticity. Videos topics include: The importance of safe sex, Discussing STIs, Health service costs, and Sex and the law.

Conclusion The videos were promoted and have been well received by a number of tertiary education institutes, youth and student organisations and sexual health agencies. The videos were promoted via social media, through relevant websites, newsletters and distributing promotional cards and posters. Conclusions on the effectiveness of the videos will be discussed once preliminary evaluation data is obtained from Google Analytics and the target audience.

Disclosure of interest statement This paper is supported and funded by the WA Department of Health.

\section{P03.22 INFECTION PROTECTION: THE DEVELOPMENT OF AN INTERACTIVE, ONLINE GAME TO EDUCATE YOUNG PEOPLE ABOUT SEXUAL HEALTH AND RELATIONSHIPS}

M Roberts*, K Kerry. Communicable Disease Control Directorate, Department of Health WA

10.1136/sextrans-2015-052270.250

Background Get the Facts (GTF) is a website which provides information on sexual health and relationships to young people aged 13-17 years. In 2014, GTF ran a game design competition which resulted in the addition of an innovative sexual health education game titled Infection Protection added to the website. The use of games for learning is recognised as an innovative and engaging method of communicating important information to young people. It provided the opportunity for young people to deliver sexual health education to their peers using an interactive and engaging format. 